\title{
Matériaux et constructions. Sciences et techniques des matériaux, de la carrière à la mise en œuvre : bilan et perspectives
}

\section{Stéphane Büttner}

\section{(2) OpenEdition \\ 1 Journals}

\section{Édition électronique}

URL : https://journals.openedition.org/cem/6722

DOI : $10.4000 /$ cem. 6722

ISSN : 1954-3093

Éditeur

Centre d'études médiévales Saint-Germain d'Auxerre

\section{Édition imprimée}

Date de publication : 15 août 2008

ISSN : 1623-5770

Référence électronique

Stéphane Büttner, « Matériaux et constructions. Sciences et techniques des matériaux, de la carrière à la mise en œuvre : bilan et perspectives », Bulletin du centre d'études médiévales d'Auxerre / BUCEMA [En ligne], 12 | 2008, mis en ligne le 27 août 2009, consulté le 22 septembre 2022. URL : http:// journals.openedition.org/cem/6722 ; DOI : https://doi.org/10.4000/cem.6722

Ce document a été généré automatiquement le 22 septembre 2022.

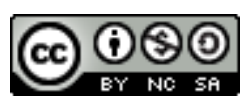

Creative Commons - Attribution - Pas d'Utilisation Commerciale - Partage dans les Mêmes Conditions 4.0 International - CC BY-NC-SA 4.0

https://creativecommons.org/licenses/by-nc-sa/4.0/ 


\title{
Matériaux et constructions. Sciences et techniques des matériaux, de la carrière à la mise en œuvre : bilan et perspectives
}

\author{
Stéphane Büttner
}

En novembre 2007, s'est achevé un post-doctorat réalisé sous la codirection de Christian Sapin et de Jean Rosen. Ces deux années ont été mises à profit pour répondre à la thématique énoncée; à savoir alimenter certains programmes de recherche développés par les équipes «études médiévales ${ }^{1}$ " et "anthropisation de l'environnement ${ }^{2}$ » de l'UMR ARTeHIS, en particulier sur les terres cuites architecturales, les liants de maçonnerie - mortier de tuileau plus spécifiquement - et la pierre à bâtir ${ }^{3}$. Cette période a aussi été mise à profit pour valoriser ces travaux par le biais de publications et de présentations lors de colloques internationaux.

\section{Les terres cuites architecturales médiévales}

2 Cette problématique avait été engagée dès 2003 dans le cadre d'une prospection thématique Approches des productions de terres cuites architecturales dans l'Yonne du Moyen Âge à la révolution industrielle, $V^{e}$-XIXe siècle (2003-2004) sous la direction de Sylvain Aumard. Dans la continuité de ces travaux, concernant plus spécifiquement les tuiles médiévales, nous avons pu démontrer, sur le corpus choisi, que la typologie établie à partir de critères morphologiques pouvait trouver une réponse lors de l'analyse pétrographique des pâtes - cathédrales d'Auxerre, de Sens, église de Noyers, abbaye de Pontigny... Outre l'observation macroscopique, qui permet déjà de révéler la nature de la cuisson (oxydante ou réductrice) et la nature de certains constituants, l'étude a été réalisée au microscope photonique polarisant. Cette échelle d'observation a permis de mettre en évidence ou de préciser la nature de certains composants que sont les dégraissants ajoutés (sable ou chamotte) ou les inclusions minérales présentes 
naturellement dans les argiles (quartz et/ou feldspaths). Mais c'est surtout les critères de forme qui paraissent caractéristiques de certaines productions; ainsi, les effets de feuilletages, souvent soulignés par la disposition des minéraux, semblent être un indice d'origine anthropique, puisque sans doute issus de la préparation avant cuisson pressage de l'argile dans le moule. Certaines productions vont d'ailleurs se caractériser par l'absence de ces formes.

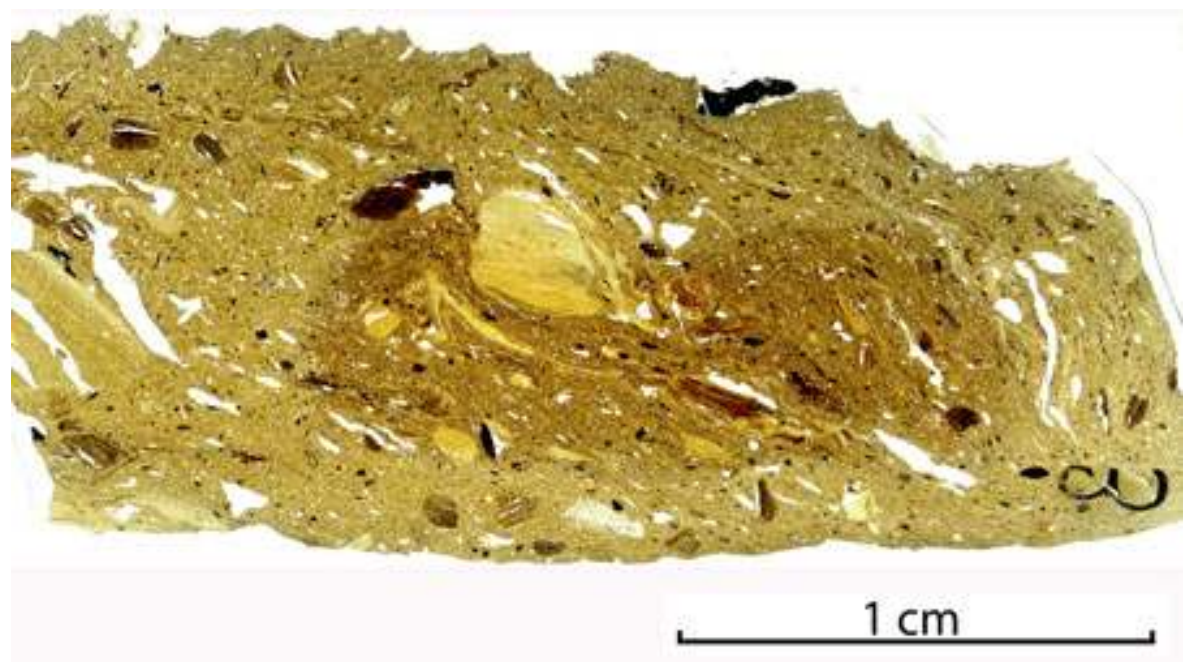

Fig 1 - Lame mince réalisée sur une tuile de l'église de Noyers-sur-Serein (éch. NOY36 - Tuile type A7 - Lumière naturelle). Texture rubanée soulignée par la disposition des oxydes et des éléments de chamottes ajoutés.

3 Il a été également envisagé d'établir un lien entre les produits finis et les centres de production probables. Pour ce faire, différentes approches ont été engagées. Plusieurs analyses archéométriques ont été ainsi produites sur une partie du corpus. Outre les analyses par thermoluminescence ou archéomagnétisme, qui ont permis de dater certains lots et ainsi d'en conforter l'homogénéité pressentie, beaucoup d'espoir ont été placés dans la diffraction X et la fluorescence X. Pour le moment, cette entreprise, longue à mettre en œuvre, n'a été réalisée que sur un nombre réduit d'individus. Pourtant, ces analyses permettent d'ores et déjà de conforter les typologies évoquées, voire d'envisager des rapprochements entre différents sites. Mais le nombre réduit d'analyses, et surtout le manque d'études comparatives sur des argiles prélevées en contexte naturel, ne permet pas d'établir de lien direct avec des argilières et donc de situer clairement les zones de production. L'autre démarche, conjointe à la précédente, consistait à compulser la documentation concernant le sujet, qu'ils s'agisse des sources écrites anciennes ou de la documentation géologique - cartes géologiques, analyses réalisées sur les argiles naturelles, etc. Si ces approches couplées ont pu permettre de situer certains centres de production potentiels, elles ont surtout permis de mettre en évidence que les tuiles sont des produits, sans doute, largement composites, pouvant associer plusieurs argiles et qui sont très souvent l'objet d'ajouts (sable ou chamotte), « masquant » à l'analyse la nature des matériaux originels utilisés pour leur confection.

4 Ces travaux ont participé à appuyer la demande du Centre d'études médiévales d'Auxerre ${ }^{4}$ et permis d'obtenir un financement PNRC sur deux ans (2008-2010) ${ }^{5}$. Celuici permettra de réaliser datations et analyses de pâte sur l'ensemble du corpus récolté dans la vallée de l'Yonne (environ 1000 échantillons). 
5 Le problème de la datation des terres cuites architecturales a été également abordé au sein du GDR européen Terres cuites architecturales et nouvelles méthodes de datation. Si les objets étudiés dans ce cadre n'ont pas fait l'objet d'analyse pétrographique, c'est surtout l'étude du bâti associé à ces productions, mais aussi l'analyse des mortiers qui ont motivé notre participation (cf. infra) ${ }^{6}$.

\section{Le mortier dans la construction médiévale}

Ces deux années ont permis d'élargir le corpus des mortiers médiévaux dans la lignée de ce qui avait été déjà entrepris - établissement de typochronologies de sites selon des critères pétrographiques, étude de la fonction architecturale. Le développement de ce corpus avait pour objectif d'améliorer les connaissances actuelles sur la fabrication et l'emploi de ce matériau au Moyen Âge: choix techniques, évolutions techniques, influences environnementales. Plusieurs édifices ont ainsi fait l'objet de telles analyses : la cathédrale Saint-Étienne d'Auxerre et l'abbaye de Pontigny (Yonne), le donjon de la Marche (Nièvre), l'église de Saint-Aubin (Côte-d'Or), l'abbaye de Cluny (Saône-et-Loire), l'église de Brienne-la-Vieille (Aube), l'église Notre-Dame-du-Port à Clermont (Puy-deDôme), Notre-Dame-sous-Terre au Mont-Saint-Michel (Manche), l'église de SaintAndré-des-Eaux (Côtes-d'Armor), l'église de Saint-Lupicin (Jura), la crypte de SaintQuentin (Aisne), et le baptistère de Byllis (Albanie). Enfin, à l'instar des études menées sur les mortiers et les enduits de la grange cistercienne de Semmadon (Haute-Saône) ${ }^{7}$, des investigations ont aussi été entreprises sur tout un ensemble de granges appartenant à l'abbaye de Pontigny. Cette approche a permis de montrer certains traitements spécifiques propres aux maçonneries de ces bâtiments médiévaux à vocation agricole.

7 L'ensemble de ces investigations viendra pour partie alimenter un ouvrage sur les liants de maçonnerie, qui reste actuellement en préparation ${ }^{8}$.

8 À ce titre, dans le cadre du PCR Matériaux, techniques de construction et datation entre Loire et Saône autour de l'an Mil, un axe de recherche spécifique a été développé sur l'usage des mortiers contenant du tuileau au Moyen Âge, qu'il s'agisse de liant de maçonnerie ou de préparation de sol - en chape ou liant des tesselles de mosaïque. Si l'utilisation du mortier de tuileau dans ces fonctions semble être effectivement continu de l'Antiquité au XII ${ }^{\mathrm{e}}$ siècle, il semble connaître une inflexion de l'Antiquité tardive à l'époque carolingienne, avec, par la suite, un usage quasi systématique dans les structures de fondation de certains édifices romans du centre de la France - Saint-Aignan d'Orléans, abbaye de Marmoutier à Tours, cryptes des cathédrales Saint-Étienne d'Auxerre et Notre-Dame de Chartres, etc. Ces investigations montrent que ce mortier de tuileau est sans doute un des éléments clefs pouvant alimenter la réflexion sur la continuité des savoir-faire techniques à la transition entre l'Antiquité et le Moyen Âge ; cette réflexion sera prochainement présentée lors du colloque international Décors et espace architectural en Gaule entre l'Antiquité et le haut Moyen Âge 9.

9 Toujours en lien avec cette problématique, la participation au GDRE Terres cuites architecturales et nouvelles méthodes de datation a permis également d'ouvrir de nouvelles pistes de réflexion quant à la datation de ces mortiers. Ainsi, en collaboration avec le CRPAA de Bordeaux, des datations par thermoluminescence des tuileaux contenus dans les mortiers ont été envisagées à moyen terme ${ }^{10}$. Cette démarche devrait permettre de vérifier s'il y a perdurance des préceptes énoncés par Vitruve, qui conseille d'utiliser 
des fragments de tuiles anciennes pour la préparation de ces mortiers hydrauliques ${ }^{11}$. De même, plusieurs méthodes de datation sur les structures maçonnées d'un même édifice ayant pu être produites dans le cadre de ce groupe de recherche - Notre-Damesous-Terre du Mont-Saint-Michel en particulier -, il est également apparu que les datations radiocarbones obtenues sur les charbons de bois contenus dans les mortiers sont possiblement plus anciennes que celles réalisées par thermoluminescence sur les terres cuites de ces mêmes édifices - entre 100 à 200 ans de décalage ${ }^{12}$. Ceci ouvre toute une réflexion sur ce phénomène "vieux bois", montrant qu'il faut alors éventuellement pondérer les datations radiocarbones sur les charbons de bois récoltés dans les mortiers.

\section{Production et utilisation des pierres bourguignonnes de l'Antiquité à l'époque moderne}

L'activité essentielle produite dans le cadre de ce thème a consisté dans l'élaboration d'une base de données « Archéomatériaux » recensant les différents types de roches en œuvre dans le bâti ancien bourguignon, de l'Antiquité à l'époque moderne, l'objectif étant d'aboutir à une vision spatio-temporelle de l'usage des matériaux, tant du point de vue de leur nature intrinsèque que de leurs caractéristiques techniques. Cette base recense non seulement les matériaux en œuvre - position architecturale, caractéristiques techniques, chronologie -, mais aussi les sites d'extraction probables ou avérés, en intégrant un ensemble de données archéologiques, archéométriques et textuelles. Le projet a associé plusieurs chercheurs de l'UMR ARTeHIS $5594{ }^{13}$, des membres de l'UFR Sciences de la terre de l'université de Bourgogne ${ }^{14}$, ainsi que des institutions telles que le BRGM ${ }^{15}$ et le LRMH ${ }^{16}$.

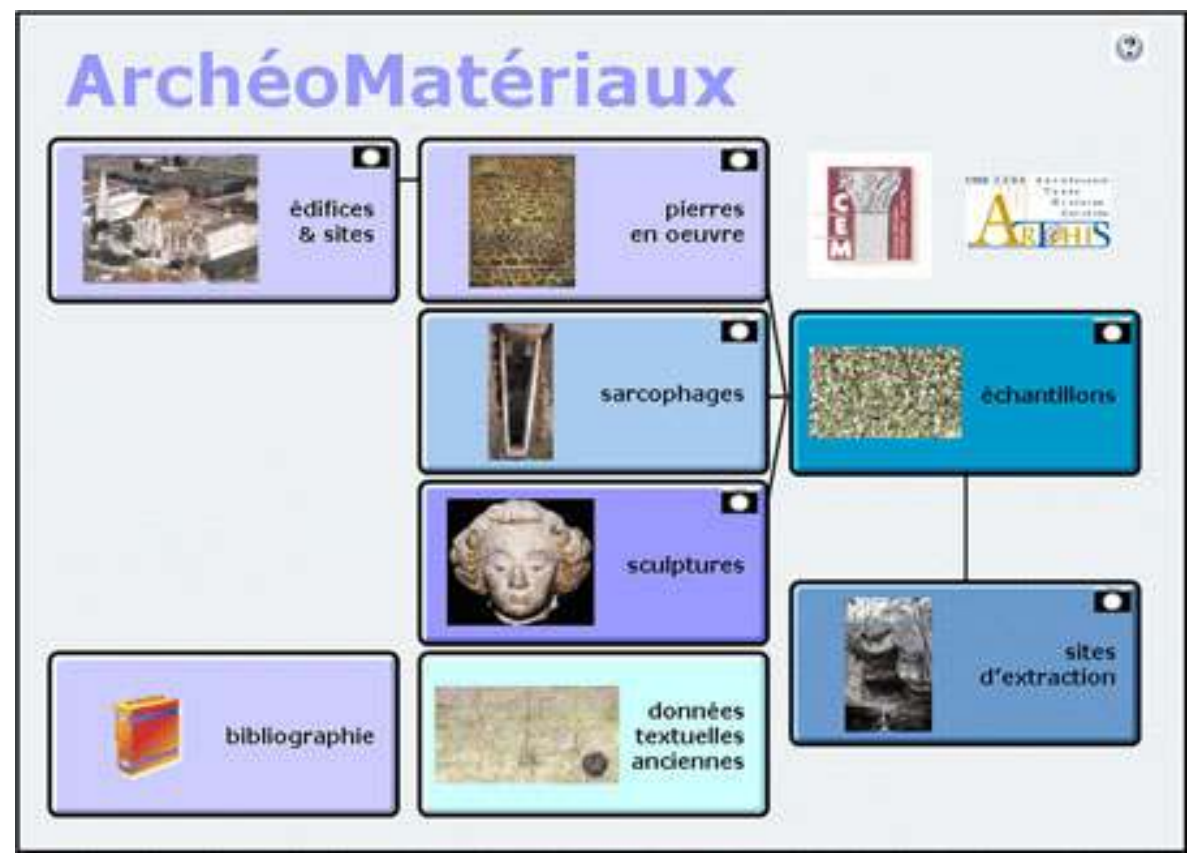

Fig. 2 - Page d'accueil de la base « Archéomatériaux ».

11 L'outil est pour le moment en phase d'essai et a été alimenté par quelques recherches entreprises sur les monuments médiévaux de la Bourgogne du Nord et de la bordure sud-ouest du Bassin de Paris ${ }^{17}$. La base de données a été également nourrie par des 
mémoires du master II Archéosciences de l'université de Bourgogne, codirigés dans le cadre de ce post-doctorat, et traitant de faciès bourguignons spécifiques et réputés ${ }^{18}$. Enfin, la nécessité d'ouvrir cette base à d'autres corpus est apparue rapidement comme une nécessité. L'ajout à la base de données de modules concernant la sculpture ou bien les sarcophages du haut Moyen Âge est apparu comme véritablement pertinent quant aux problématiques fixées ${ }^{19}$.

12 L'intérêt et l'apport d'une telle démarche seront prochainement exposés lors du premier Congrès francophone d'histoire de la construction qui se tiendra à Paris au mois de juin ${ }^{20}$. Cette présentation démontrera l'intérêt que peut avoir cette approche, quant à une meilleure connaissance de l'économie des chantiers de construction du Moyen Âge.

L'ensemble de ces démarches a alimenté la réflexion concernant les techniques de construction et l'évolution des savoir-faire au Moyen Âge. Elle implique aussi toute une réflexion sur l'économie du minéral au Moyen Âge. Ces investigations ont été, pour partie, présentées dans le cadre de colloques internationaux et ont fait l'objet d'articles ${ }^{21}$. Il apparaît également que ces thèmes de recherche n'en sont qu'à leurs balbutiements. Malgré la production scientifique effective sur ces sujets, il semble aujourd'hui que c'est surtout un effort de synthèse qu'il faut produire. Pour ce faire, le projet de base de données "Archéomatériaux » devrait prochainement connaître un développement supplémentaire, en lui associant les données liées aux terres cuites architecturales. À terme, l'intention est d'intégrer l'ensemble de ces données à un système d'informations géoréférencées (SIG), l'objectif étant d'aboutir à l'établissement d'un véritable "Atlas des matériaux et des techniques du bâti médiéval », certain de l'apport qu'un tel projet pourrait avoir dans les domaines de l'archéologie du bâti, de l'histoire de l'architecture et de l'histoire de l'art.

\section{INDEX}

Mots-clés : matériau, mortier, pierre bourguignonne, TCA (Terres cuites architecturales) 